\title{
Cisplatin regulates SH-SY5Y cell growth through downregulation of BDNF via miR-16
}

\author{
YUN-XIAO SUN ${ }^{1,2}$, JIAN YANG $^{3}$, PING-YU WANG ${ }^{4}$, YOU-JIE LI ${ }^{4}$, SHU-YANG XIE $^{1,4}$ and RUO-PENG SUN ${ }^{1}$ \\ ${ }^{1}$ Department of Pediatrics, Qilu Hospital, Shandong University, Jinan 250012; ${ }^{2}$ Department of Pediatrics, \\ Binzhou Medical University, Yantai 264003; ${ }^{3}$ Yantai Central Blood Station, Yantai 264003; \\ ${ }^{4}$ Key Laboratory of Tumor Molecular Biology, Department of Biochemistry and Molecular Biology, \\ Binzhou Medical University, Yantai 264003, P.R. China
}

Received July 12, 2013; Accepted August 23, 2013

DOI: $10.3892 /$ or.2013.2731

\begin{abstract}
Brain-derived neurotropic factor (BDNF) is a member of the neurotropin family. High levels of BDNF are associated with more aggressive malignant behavior in human cancer. In the present study, we observed the effect of cisplatin on BDNF expression in SH-SY5Y cells and investigated the mechanism of cisplatin in inducing the apoptosis of SH-SY5Y cells. Our results revealed that the expression of BDNF was obviously decreased in cisplatin-treated SH-SY5Y cells. In addition, the 3'-untranslated region of BDNF was found to be targeted by miR-16 using microRNA analysis software. After miR-16 was synthesized chemically, SH-SY5Y cells were transfected with miR-16 to investigate the regulatory role of miR-16 in regards to BDNF. The results showed that the expression of BDNF was markedly decreased in the miR-16-transfected cells when compared with that in the control cultures as determined by western blotting. Moreover, miR-16 expression was obviously upregulated in the cisplatin-treated cells when compared with the untreated controls. Furthermore, SH-SY5Y cells were xenografted subcutaneously in nude mice to study the effect of cisplatin on the growth of SH-SY5Y cells in vivo. The results
\end{abstract}

Correspondence to: Professor Ruo-Peng Sun, Department of Pediatrics, Qilu Hospital, Shandong University, 107 West Wenhua Road, Jinan 250012, P.R. China

E-mail: luckysrp@163.com

Professor Shu-Yang Xie, Key Laboratory of Tumor Molecular Biology, Department of Biochemistry and Molecular Biology, Binzhou Medical University, 346 Guanhai Road, Yantai 264003, P.R. China

E-mail: shuyangxie@aliyun.com

Abbreviations: miRNAs, microRNAs; BDNF, brain-derived neurotropic factor; 3'-UTR, 3'-untranslated region; TrkB, tyrosine kinase receptor B; MTT, 3-(4,5-dimethylthiazol-2-yl)-2,5-diphenyltetrazolium bromide; DMSO, dimethyl sulfoxide; FACS, flow cytometry; HRP, horseradish peroxidase; AD, Alzheimer disease

Key words: microRNAs, neuroblastoma, BDNF, cisplatin, cell proliferation, gene expression further showed that cisplatin inhibited the proliferation of SH-SY5Y cells in the cisplatin-treated mice when compared with the saline-treated control. The expression of miR-16 was increased, while the expression of BDNF was decreased in the cisplatin-treated mice. Our results demonstrated that cisplatin downregulated the expression of BDNF through miR-16 to inhibit SH-SY5Y cell proliferation in vitro and in vivo. These findings provide the basis for new targets for drug design or cancer therapy.

\section{Introduction}

Neuroblastoma is one of the most common childhood tumors, usually diagnosed at a median age of 17 months (1). The tumor originates from neural-crest tissues, deep in the adrenal medulla and paraspinal ganglia, with no specific clinical presentations (2). Approximately 50-60\% patients are diagnosed with neuroblastoma at advanced stages (3). In spite of multidisciplinary care, even the introduction of dose intensive chemotherapy (4), the outcome of high risk neuroblastoma has achieved no significant improvement (5), with a 5-year survival rate of $<50 \%$ (6).

Brain-derived neurotropic factor (BDNF), a member of the neurotropin family, plays a critical role in neuronal survival, differentiation and axon wiring through binding to its preferred receptor TrkB (tyrosine kinase receptor B) (7-9). Research has demonstrated that BDNF-TrkB signals do not only regulate the growth of nerve neutrons, but also affect the development, invasion and outcome of many types of tumors, including lung (10), bladder (11) and pancreatic cancer (12). A recent study showed that BDNF enhanced the proliferation and survival of transitional cell carcinoma (13). High expression levels of BDNF are associated with more aggressive malignant behavior in human cancers, including pancreatic (14) and breast cancer (15).

microRNAs (miRNAs) are small non-coding RNAs, involved in the post-transcriptional regulation of gene expression. Through binding to the imperfect sequence of target mRNAs, miRNAs result in translational inhibition or destabilization of target mRNAs. miRNAs can act as tumor suppressors or as oncogenes (16). miR-106a was shown to be upregulated in gastric cancer, having pro-tumorigenic 
effects (17). miR-16 functions as a tumor suppressor and was found to be downregulated in mantle cell lymphoma SP cells by regulating Bmil, leading to reduction in tumor size in lymphoma xenografts (18).

Cisplatin is an effective drug in the in vivo treatment of neuroblastoma. It also can inhibit the growth of neuroblastoma cells and reduce cell viability (19). In our previous study, we found that miR-21 expression was decreased after treatment with cisplatin, while MSH2 expression was enhanced (20). In the present study, we further explored the mechanism of cisplatin in suppressing the proliferation of neuroblastoma cells and found that cisplatin downregulated the expression of BDNF through upregulation of miR-16 to inhibit SH-SY5Y cell proliferation.

\section{Materials and methods}

Cell culture. The neuroblastoma cell line SH-SY5Y, obtained from the Shanghai Institute of Biochemistry and Cell Biology (Shanghai, China), was cultured in DMEM/F12 (1:1) (HyCLone, Logan, UT, USA) with 10\% FCS (HyClone), $100 \mathrm{U} / \mathrm{ml}$ penicillin and $100 \mu \mathrm{g} / \mathrm{ml}$ streptomycin at $37^{\circ} \mathrm{C}$ in $5 \%$ $\mathrm{CO}_{2}$. Cells were detached from cell culture flasks with $0.25 \%$ trypsin when they grew and spread in the bottom of the flasks (at $48 \mathrm{~h}$ ) and were subcultured in fresh culture medium.

Cisplatin treatment. SH-SY5Y cells in logarithmic phase were detached, counted and planted into cell culture plates. On the following day, different concentrations of cisplatin $(0$, 1.5, 3, 4.5, 6 and $12 \mu \mathrm{g} / \mathrm{ml}$, separately) (QiLu Pharmaceutical Co., Ltd., Jinan, China) were dissolved in the culture medium. Cells were collected for subsequent experiments at $48 \mathrm{~h}$ after cisplatin treatment.

MTT detection. SH-SY5Y cells were planted into a 96-well plate at a density of $1 \times 10^{4}$ cells/well. The following day, each group was treated with corresponding concentrations of cisplatin according to the experimental design. MTT $(10 \mu \mathrm{l})$ (5 mg/ml; Sigma, St. Louis, MO, USA) was added into each well after $48 \mathrm{~h}$. Then, $100 \mu \mathrm{l}$ DMSO (Sigma) was added to each well. The plate was shaken until the MTT was fully dissolved. After that, the OD value was measured with an enzyme-linked immunosorbent assay reader (ELx800, USA) at $570 \mathrm{~nm}$. Cell growth inhibition rate was calculated as follows: Cell growth inhibition rate $=\left(\mathrm{OD}_{\text {control }}-\mathrm{OD}_{\text {sample }}\right) / \mathrm{OD}_{\text {control }} \times 100(\%)$.

Flow cytometric analysis. SH-SY5Y cells $\left(8 \times 10^{4}\right)$ were treated with cisplatin for $48 \mathrm{~h}$, the culture medium was discarded, and the SH-SY5Y cells were collected. Binding buffer $(500 \mu \mathrm{l})$ (KeyGen Biotech Co., Ltd., Nanjing, China) was added to the collected cells to suspend the cells. Annexin V-FITC $(5 \mu 1)$ was added with gently mixing. After that, $5 \mu \mathrm{l}$ PI was added to the cells. The cells were incubated for $15 \mathrm{~min}$ and measured by flow cytometry (Beckman-Coulter, Inc., Brea, CA, USA) with a 488-nm exciting wavelength and a 530-nm emitting wavelength.

miRNA transfection. miR-16 and NC (control oligos) were chemically synthesized (GenePharma Co., Ltd., Shanghai, China). The sense sequence of the miR-16 was 5'-UAGCAGCA
CGUAAAUAUUGGCG-3'; the antisense was 5'-CCAAUAUU UACGUGCUGCUAUU-3'. The sense sequence of NC was 5'-CAGUACUUUUGUGUAGUACAA-3'; the antisense was 5'-GUACUACACAAAAGUACUGUU-3'. The cells were treated with $1 \mu \mathrm{g}$ oligos and $2.5 \mu \mathrm{l}$ Lipofectamine 2000 (Invitrogen, Carlsbad, CA, USA) as introductions. Cells were collected at $48 \mathrm{~h}$ after transfection.

SH-SY5Y cell xenografts and cisplatin interference. BALB/C-nu mice (nude mice) were obtained from HFK Bio-Technology Co., Ltd. (Beijing, China). The nude mice were narcotized with $1.5 \%$ pentobarbital sodium and were injected subcutaneously with $1 \times 10^{7}$ SH-SY5Y cells. Tumors appeared 3 days after cell xenograft. When the tumor volume ( $\mathrm{V}=$ length $\mathrm{x}$ width $2 / 2$, length $>$ width) grew to $100 \mathrm{~mm}^{3}$, the mice were treated with $3 \mathrm{mg} / \mathrm{kg}$ cisplatin by intraperitoneal injection once every 4 days, the treatment was carried out 4 times all together. The control group was injected with the same volume of saline solution. After the last treatment, the mice were sacrificed to assess the tumors. The tumor weights were determined with an electronic scale. Subsequently, the tumor tissues were analyzed for the levels of miRNA or BDNF protein.

Western blotting. SH-SY5Y cells and xenograft tumors were collected and lysed to extract the total protein following cisplatin treatment. Protein $(40 \mu \mathrm{g})$ of each sample was loaded respectively on each lane of a polyacrylamide gel. Protein bands on the separating gel were transferred to a PVDF membrane through a transfer device. Then, rabbit anti-BDNF polyclonal antibody (1:200; Santa Cruz Biotechnology, Santa Cruz, CA, USA) was added to the membrane for overnight incubation at $4^{\circ} \mathrm{C}$. The following day, the membrane was incubated in HRP-labeled goat anti-rabbit IgG for $2 \mathrm{~h}(1: 6,000$; Beijing Zhongshan Golden Bridge Technology Co., Ltd., Beijing, China). Finally, a chemical spectral imager (Tanon, China) was applied to observe the results.

Real-time PCR. RNAiso for small RNA (Takara, Shiga, Japan) was applied to extract miRNA from SH-SY5Y cells or xenografts. Poly(A) was added using poly(A) polymerase (Ambion, Foster City, CA, USA). The cDNA was synthesized by RT primer 5'-AACATGTACAGTCCATGGATGd(T) ${ }_{30} \mathrm{~N}(\mathrm{~A}, \mathrm{G}, \mathrm{C}$ or T)-3'. The forward primer used to amplify miR-16 was 5'-TAGCAGCACATAAATATTGGCG-3' and the reverse was 5'-AACATGTACAGTCCATGGATG-3'. Forward primer of 5S rRNA was 5'-GCCATACCACCCTGAACG-3' and the reverse was 5'-AACATGTACAGTCCATGGATG-3'. SYBR ${ }^{\circledR}$ Premix Ex Taq ${ }^{\mathrm{TM}}$ kit (Takara) is used to conduct qRT-PCR. The expression of miR-16 was assessed using the RG3000 system (Corbett Research, Sydney, Australia). Denaturing was carried out at $95^{\circ} \mathrm{C}$ for $3 \mathrm{~min} ; 40$ cycles of $95^{\circ} \mathrm{C}$ for $20 \mathrm{sec}$; annealing at $60^{\circ} \mathrm{C}$ for $20 \mathrm{sec}$ and extension at $72^{\circ} \mathrm{C}$ for $20 \mathrm{sec}$. At each extension step at $72^{\circ} \mathrm{C}$, fluorescence was detected at $585 \mathrm{~nm}$. The human $5 \mathrm{~S}$ rRNA served as the control.

Statistics. SAS software was used to analyze the significance of all results. The Student's t-test was used for inter-group comparison. A P-value $<0.05$ was considered to indicate a statistically significant result. 


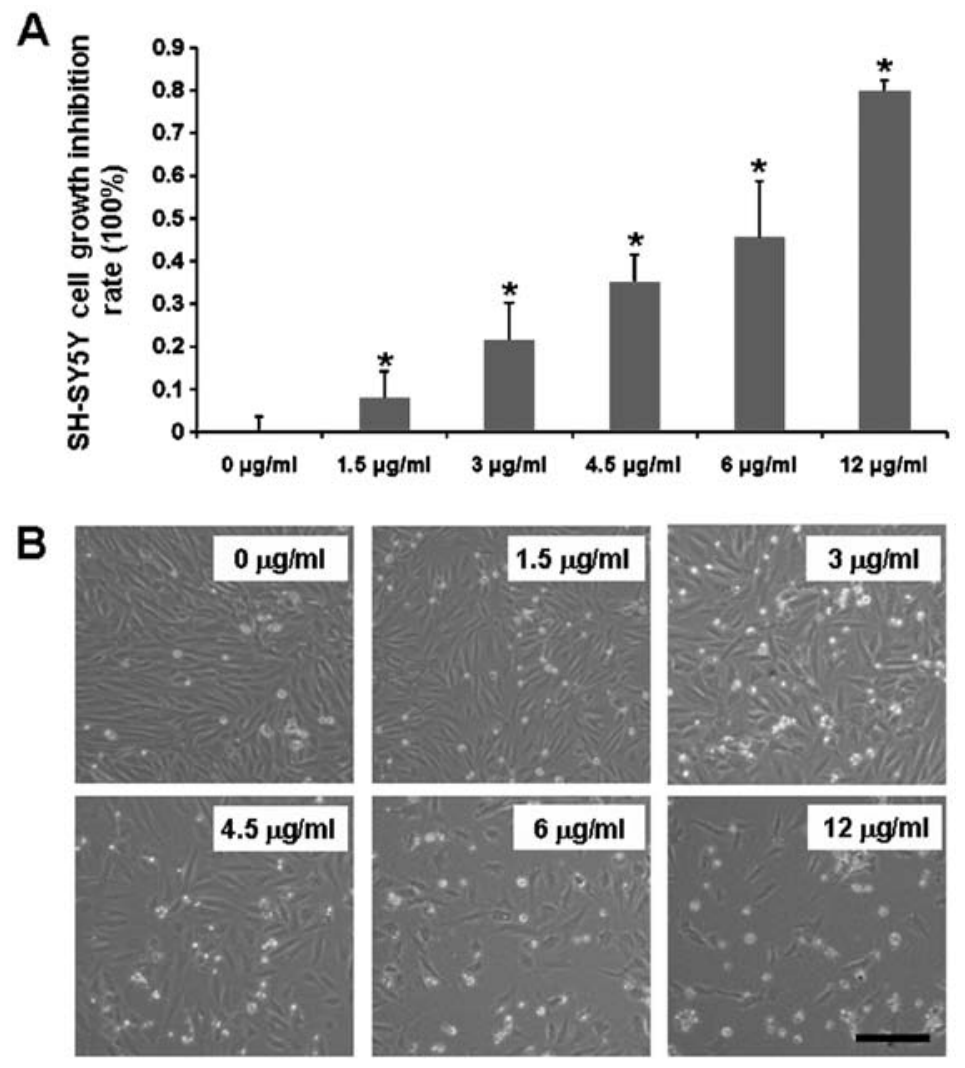

Figure 1. Cisplatin inhibits SH-SY5Y cell growth. (A) MTT assay revealed that the growth inhibition rate of SH-SY5Y cells was increased with the higher concentrations of cisplatin. ${ }^{*} \mathrm{P}<0.05$ vs. control $(0 \mu \mathrm{g} / \mathrm{ml})$ cells. (B) As detected under an inverted microscope, a fewer number of live cells was noted following treatment with increasing concentrations of cisplatin. Scale bar, $100 \mu \mathrm{m}$.
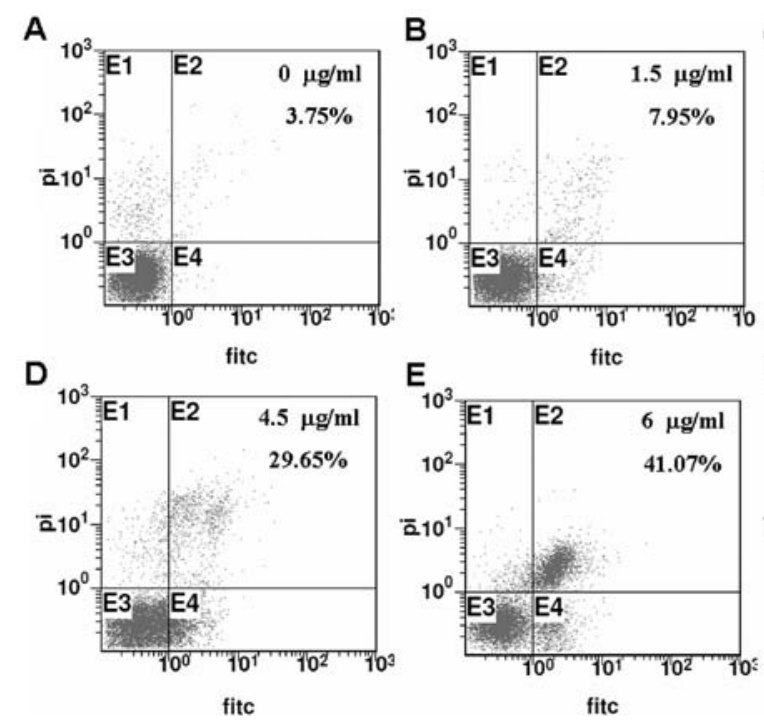

fitc
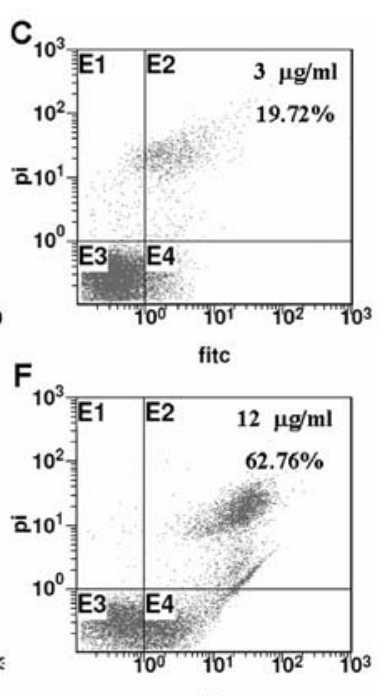

fitc

Figure 2. Analysis of apoptotic cells by FACS. The results showed that the percentage of apoptotic cells was increased after cisplatin treatment. The effect was more obvious following treatment with cisplatin at concentrations $\geq 6 \mu \mathrm{g} / \mathrm{ml}$. (A-E) The apoptotic percentages of SH-SY5Y cells was detected by FACS after 0, $1.5,3,4.5,6$ and $12 \mu \mathrm{g} / \mathrm{ml}$ cisplatin treatment, separately.

\section{Results}

Cisplatin inhibits the proliferation of SH-SY5Y cells. To select an effective concentration of cisplatin for suppressing the proliferation of SH-SY5Y cells, an MTT assay was performed to determine the cell growth inhibition rate. The cell growth inhibition rate reached $\sim 50 \%$ in the $6 \mu \mathrm{g} / \mathrm{ml}$ cisplatin-treated cultures (Fig. 1A). The number of live cells was obviously decreased with increasing concentrations of cisplatin as noted under an inverted microscope (Fig. 1B). The percentages of apoptotic cells were also increased with increasing concentrations of cisplatin as detected by Annexin V-FITC/PI analysis (Fig. 2). These results showed that cisplatin inhibited SH-SY5Y cell proliferation significantly with increasing concentrations. 

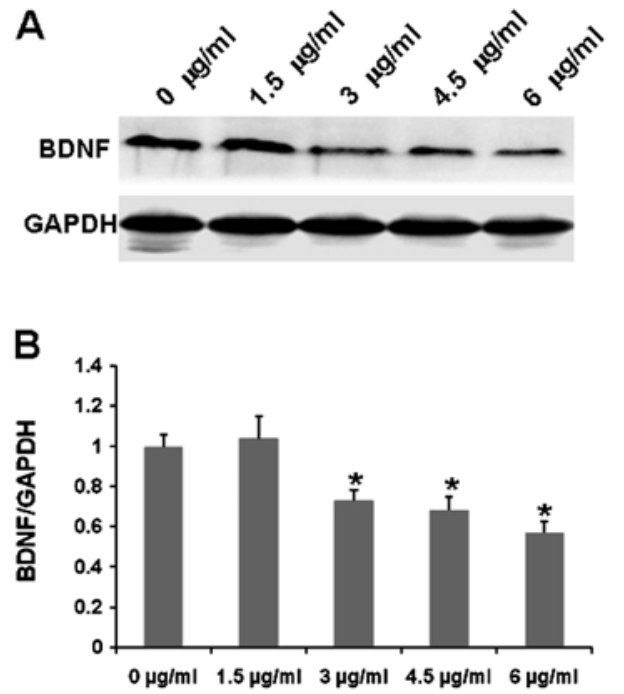

Figure 3. BDNF expression is detected by western blotting. (A) BDNF expression was decreased in the SH-SY5Y cells with increasing concentrations of cisplatin. (B) Relative values for BDNF vs. GAPDH in A. ${ }^{*} \mathrm{P}<0.05$ vs. the control $(0 \mu \mathrm{g} / \mathrm{ml})$ cells

Cisplatin decreases BDNF levels in SH-SY5Y cells. BDNF is a trophic factor for neuroblastoma cells (21). To investigate whether cisplatin inhibits SH-SY5Y cell growth by regulating BDNF levels, the BDNF expression in SH-SY5Y cells was assessed following cisplatin treatment. Western blotting showed that the expression of BDNF was obviously decreased in the cisplatin-treated cells when compared to that in the untreated controls, and the levels of BDNF were significantly reduced following treatment with increasing concentrations of cisplatin (Fig. 3). Thus, cisplatin suppresses SH-SY5Y cell growth by reducing BDNF expression.

$B D N F$ expression is regulated by miR-16. miRNAs can play roles as tumor suppressors or as oncogenes by regulating their target genes (16). Yet, few studies have focused on BDNF-related miRNAs. Based on microRNA analysis online software (http://www.targetscan.org/ or http://www.microrna. org/microrna/home.do), we found that BDNF-3'-UTR is targeted by miR-16 (Fig. 4A). Then, we synthesized miR-16 and transfected the SH-SY5Y cells with miR-16. Our results showed that the expression of BDNF was obviously decreased in the miR-16-transfected cells when compared with that in the scrambled oligos-treated cultures as determined by western blot analysis (Fig. 4B and C). These results showed that miR-16 negatively regulated BDNF expression. It is known that miR-16 can function as a tumor suppressor in mantle cell lymphoma cells (18). Then, we studied the effect of overexpression of miR-16 on SH-SY5Y cells. We found that overexpression of miR-16 inhibited SH-SY5Y cell growth and induced cell apoptosis (Fig. 5). The above findings revealed that miR-16 inhibits SH-SY5Y cell growth by negatively regulating BDNF.

Cisplatin upregulates miR-16 levels in SH-SY5Y cells. We demonstrated that BDNF is a new target of miR-16. Since cisplatin can also downregulate the expression of BDNF, we hypothesized that there may be a relationship between miR-16 expression and cisplatin treatment. To further test whether cisplatin affects miR-16 expression, real-time PCR was performed to analyze miR-16 levels following cisplatin treatment. After miRNA isolation and reverse transcription, we found that the expression level of miR-16 in cisplatintreated cells increased to a greater extent with increasing concentrations of cisplatin when compared with the untreated controls (Fig. 6). Collectively, our results showed that cisplatin regulates SH-SY5Y cell proliferation by upregulating miR-16 to inhibit BDNF in vitro.

Cisplatin inhibits growth of SH-SY5Y cell xenografts by decreasing BDNF through miR-16. To further investigate whether cisplatin inhibits SH-SY5Y cell growth in vivo, nude mice xenografted with SH-SY5Y cells were treated with cisplatin. We found that the tumor volume of SH-SY5Y cell xenografts in the cisplatin-treated nude mice was markedly reduced, and the weight of tumor xenografts was decreased when compared with these values in the saline-treated controls (Fig. 7). To study whether cisplatin also affects miR-16 or BDNF expression in xenografts, we analyzed the levels of miR-16 or BDNF by real-time PCR or western blotting. Our result showed that the expression of miR-16 was obviously

\section{A Position 299-305 of BDNF 3' UTR 5' ... AAAAUAAUAAAUUGCAUGCUGCUU... hsa-miR-16 3' 3 IIII I I IIIII}
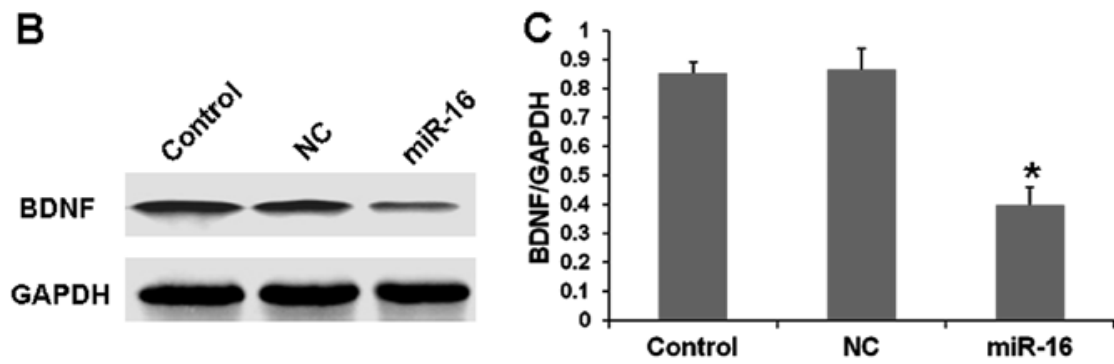

Figure 4. BDNF expression is regulated by miR-16. (A) The sequence of BDNF-3' UTR is targeted by miR-16. (B) Western blotting. The results showed that BDNF expression was decreased in the miR-16-treated cells when compared with that in the control cells. (C) Relative values for BDNF vs. GAPDH in B. ${ }^{*} \mathrm{P}<0.05$ vs. untreated control or NC-transfected cells. 

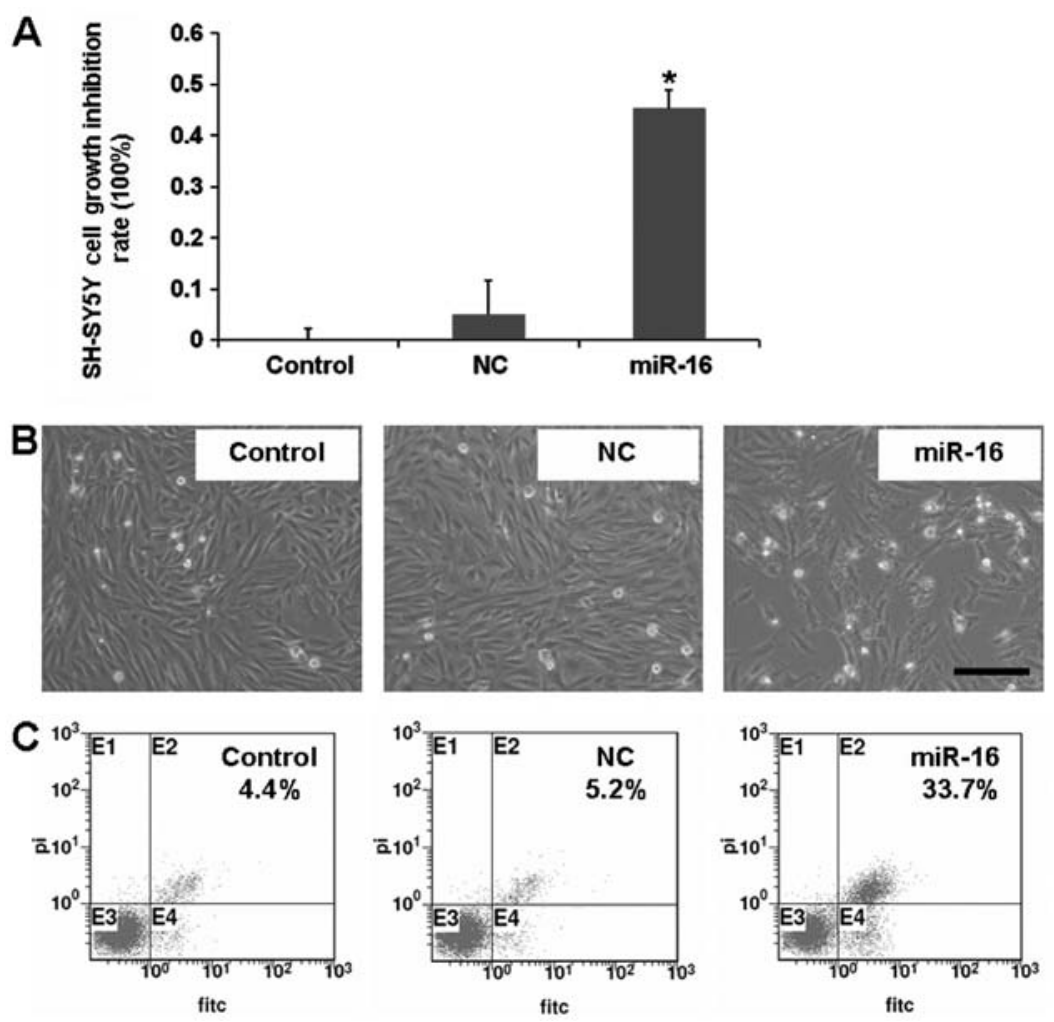

Figure 5. miR-16 regulates SH-SY5Y cell proliferation. (A) MTT assay showed that the growth inhibition rate of SH-SY5Y cells was much higher in the miR-16-transfected cells than that in the control oligo-transfected (NC) cells. ${ }^{*} \mathrm{P}<0.05$ vs. untreated control or NC-transfected cells. (B) As detected under an inverted microscope, a fewer number of live cells was noted in the miR-16-transfected cells than the number in the control cells. Scale bar, $100 \mu \mathrm{m}$. (C) FACS analysis showed that the percentage of apoptotic cells was higher in the miR-16-transfected cells when compared with the control treatment.

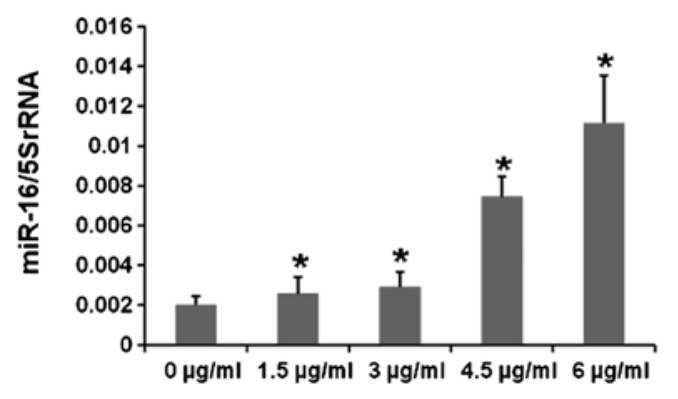

Figure 6. miR-16 expression is detected by real-time PCR. Higher levels of miR-16 were found in cisplatin-treated SH-SY5Y cells when compared with the untreated control cells. ${ }^{*} \mathrm{P}<0.05$ vs. untreated control.

increased in the cisplatin-treated cells (Fig. 8A), while BDNF levels were markedly decreased in the cisplatin-treated cells when compared with these levels in the saline-treated controls (Fig. 8B and C). Thus, cisplatin inhibits SH-SY5Y cell proliferation by upregulating miR-16 to inhibit BDNF in vivo.

\section{Discussion}

BDNF, initially identified as a neurotrophin, plays an essential role in a variety of neuronal functions, including proliferation, differentiation and survival in the central nervous system (CNS). It also contributes to the regulation of synaptic plasticity (22). In many cancer studies, the expression of BDNF was found to be correlated with tumor progression or poorer
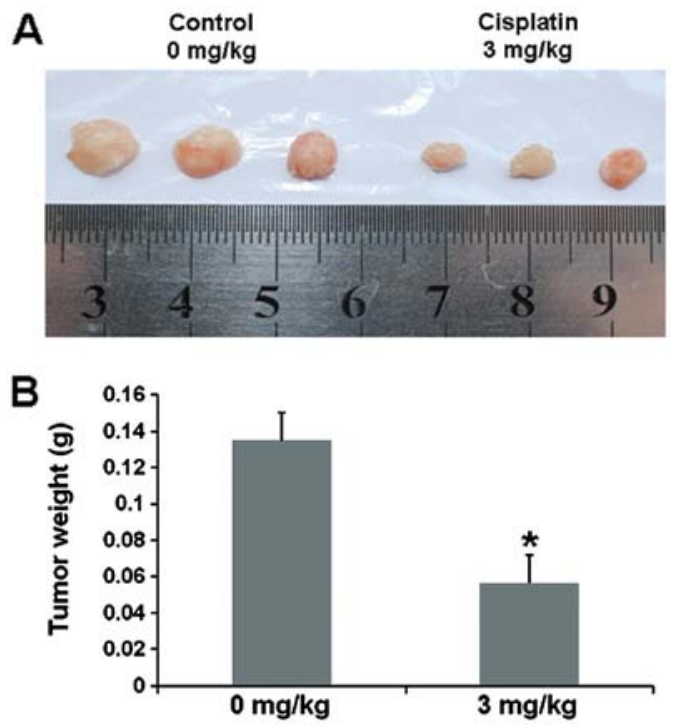

Figure 7. Cisplatin inhibits the growth of SH-SY5Y cell xenografts. (A) The tumor volume was reduced in the cisplatin-treated mice when compared with the volume in the saline-treated controls. (B) The tumor weight was reduced in the cisplatin-treated mice when compared with the tumor weight in the controls. ${ }^{*} \mathrm{P}<0.05$ vs. untreated controls.

prognosis. The increased expression of BDNF increased the risk of metastasis, regional invasion and mortality. It was found that BDNF was overexpressed in gallbladder adenocarcinoma compared with peritumoral tissues, adenoma, polyps 

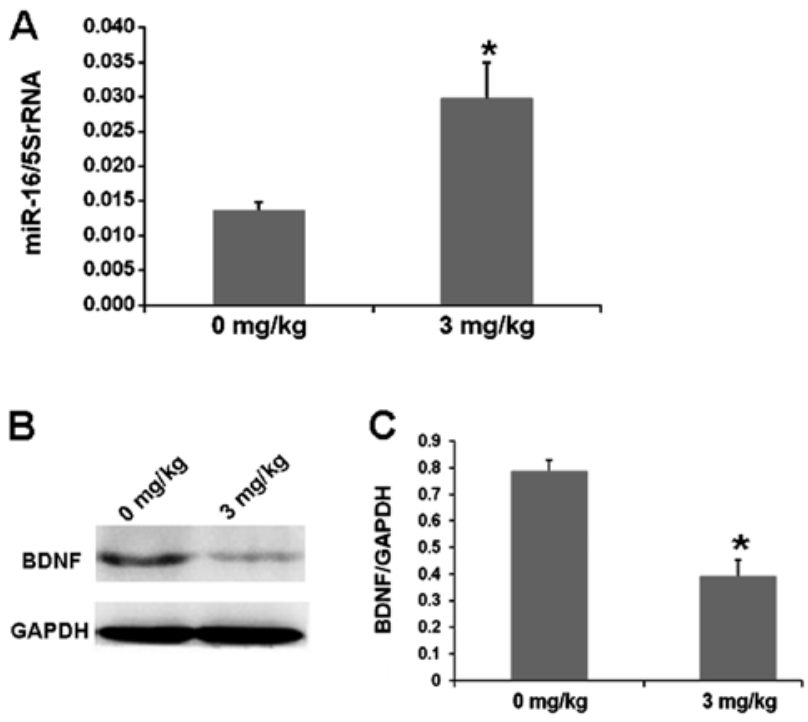

Figure 8. miR-16 and BDNF expression in SH-SY5Y cancer xenografts. (A) Real-time PCR results showed that miR-16 expression was increased in the cisplatin-treated SH-SY5Y cell xenografts when compared with that in the saline-treated controls. ${ }^{*} \mathrm{P}<0.05$ vs. saline-treated controls. (B) Western blotting demonstrated that BDNF expression was decreased in the cisplatintreated SH-SY5Y cancer xenografts in nude mice when compared with that in the saline-treated mice. (C) Relative values for BDNF vs. GAPDH in B. ${ }^{*} \mathrm{P}<0.05$ vs. the saline-treated controls.

and chronic cholecystitis samples (23). In the present study, we also found that the expression of BDNF was increased in the SH-SY5Y neuroblastoma cells, and cisplatin inhibited the proliferation of SH-SY5Y cells in vitro and in vivo by downregulating BDNF expression.

Recent studies have shown that miRNAs are involved in the initiation and progression of cancer (24). Approximately 17 differentially expressed miRNAs, including miR-339-5p, miR-423-3p, miR-19a, were reported to play important regulatory roles in the progression of lung cancer (25). miR-7 was also found to be a tumor-suppressor gene in glioblastoma, and was associated with cancer cell proliferation, invasion and metastasis (26). Overexpression of miR-7 not only induced A549 cell apoptosis and inhibited cell migration in vitro, but also reduced tumorigenicity in vivo (27). miR-16 was also found to be a tumor suppressor and was downregulated in most solid tumors, such as ovarian, prostate and colorectal cancer (28-30). Overexpression of miR-16 inhibited cell proliferation and induced cell apoptosis in CRC cells (30). In the present study, our results demonstrated that overexpression of miR-16 inhibited the proliferation of SH-SY5Y cells, which further confirmed the suppressor role of miR-16 in neuroblastoma cells.

Recent studies have shown that the expression of BDNF is regulated by miRNAs in many CNS diseases (31-33). Lee et al (31) confirmed that the expression of miR-206 was increased in AD mice and human AD brains, and bioinformatics technology revealed that BDNF is a potential target of miR-206. miR-206-neutralizing antagomir was found to increase the brain levels of BDNF and improve the memory function of $\mathrm{AD}$ mice. miR-30a-5p overexpression reduced the levels of BDNF protein in rat forebrain neurons (32). BDNF levels were also found to be a inversely correlated with
miR-195 levels in a schizophrenic group (33). Yet, few studies have revealed the mechanism of BDNF-related miRNAs in regulating tumor progression. BDNF was reported to be regulated by miR-204 in ovarian and breast cancers (34). In the present study, our results further showed that BDNF is a target of miR-16, which induced the apoptosis of SH-SY5Y neuroblastoma cells by regulating the level of BDNF in vitro and in vivo.

Cisplatin is one of the first-line chemotherapeutic drugs used for the treatment of many solid tumors $(35,36)$. Recent studies have indicated that miRNAs modulates the sensitivity to various chemotherapeutic drugs (including cisplatin) in many cancers by regulation of their target genes $(37,38)$. miR-302a expression levels were upregulated by cisplatin in a dose- and time-dependent manner in NT2 cells, and miR-302a significantly enhanced the sensitivity of NT2 cells to cisplatin through the downregulation of p21 (37). Ryan et al (38) found that upregulation of miR-204 in neuroblastoma cell lines increased the sensitivity to cisplatin and etoposide significantly by targeting NTRK 2 and BCL 2 directly. Here, we found that miR-16 was upregulated in cisplatin-treated neuroblastoma cells and xenografts, suggesting that miR-16 is a target of cisplatin, and miR-16 overexpression may enhance the anticancer effect of cisplatin. Further results demonstrated that cisplatin downregulated BDNF through reducing miR-16 in SH-SY5Y cells.

In summary, the present study demonstrated that the expression of BDNF was regulated by miR-16 in SH-SY5Y cells, and cisplatin inhibited SH-SY5Y cell proliferation in vitro and in vivo by increasing miR-16 expression and downregulating BDNF levels. These findings provide evidence for new gene targets for neuroblastoma therapy.

\section{Acknowledgements}

The present study was supported by the NCET-10-0919, National Natural Science Foundation (nos. 31371321, 81200601), the Shandong Science and Technology Committee (no. ZR2012HQ035), the Foundation of Shandong Educational Committee (nos. J10LC60, J11LC01) and the Binzhou Science and Technology Committee of China (no. 2011ZC0905).

\section{References}

1. London WB, Castleberry RP, Matthay KK, et al: Evidence for an age cutoff greater than 365 days for neuroblastoma risk group stratification in the Children's Oncology Group. J Clin Oncol 23: 6459-6465, 2005.

2. Maris JM: Recent advances in neuroblastoma. N Engl J Med 362: 2202-2211, 2010.

3. Chu CM, Rasalkar DD, Hu YJ, Cheng FW, Li CK and Chu WC: Clinical presentations and imaging findings of neuroblastoma beyond abdominal mass and a review of imaging algorithm. $\mathrm{Br} \mathbf{J}$ Radiol 84: 81-91, 2011.

4. Pavlidis N, Stahel R, Clarke M and Djulbegovic B: Cancer treatment reviews welcomes submission of the Cochrane Reviews. Cancer Treat Rev 32: 243-244, 2006.

5. Verissimo CS, Molenaar JJ, Fitzsimons CP and Vreugdenhil E: Neuroblastoma therapy: what is in the pipeline? Endocr Relat Cancer 18: R213-R231, 2011.

6. Hara J: Development of treatment strategies for advanced neuroblastoma. Int J Clin Oncol 17: 196-203, 2012.

7. Arévalo JC and Wu SH: Neurotrophin signaling: many exciting surprises! Cell Mol Life Sci 63: 1523-1537, 2006. 
8. Huang EJ and Reichardt LF: Trk receptors: roles in neuronal signal transduction. Annu Rev Biochem 72: 609-642, 2003.

9. Soppet D, Escandon E, Maragos J, et al: The neurotrophic factors brain-derived neurotrophic factor and neurotrophin-3 are ligands for the trkB tyrosine kinase receptor. Cell 65 895-903, 1991.

10. Odate S, Nakamura K, Onishi $\mathrm{H}$, et al: TrkB/BDNF signaling pathway is a potential therapeutic target for pulmonary large cell neuroendocrine carcinoma. Lung Cancer 79: 205-214, 2013.

11. Dionne CA, Camoratto AM, Jani JP, et al: Cell cycle-independent death of prostate adenocarcinoma is induced by the trk tyrosine kinase inhibitor CEP-751 (KT6587). Clin Cancer Res 4: 1887-1898, 1998

12. Miknyoczki SJ, Lang D, Huang L, Klein-Szanto AJ, Dionne CA and Ruggeri BA: Neurotrophins and Trk receptors in human pancreatic ductal adenocarcinoma: expression patterns and effects on in vitro invasive behavior. Int J Cancer 81: 417-427, 1999.

13. Huang YT, Lai PC, Wu CC, et al: BDNF mediated TrkB activation is a survival signal for transitional cell carcinoma cells. Int J Oncol 36: 1469-1476, 2010

14. Sclabas GM, Fujioka S, Schmidt C, et al: Overexpression of tropomysin-related kinase B in metastatic human pancreatic cancer cells. Clin Cancer Res 11: 440-449, 2005.

15. Patani N, Jiang WG and Mokbel K: Brain-derived neurotrophic factor expression predicts adverse pathological and clinical outcomes in human breast cancer. Cancer Cell Int 11: 23, 2011.

16. Chen PS, Su JL and Hung MC: Dysregulation of microRNAs in cancer. J Biomed Sci 19: 90, 2012.

17. Zhou H, Guo JM, Lou YR, et al: Detection of circulating tumor cells in peripheral blood from patients with gastric cancer using microRNA as a marker. J Mol Med 88: 709-717, 2010.

18. Teshima K, Nara M, Watanabe A, et al: Dysregulation of BMI1 and microRNA-16 collaborate to enhance an anti-apoptotic potential in the side population of refractory mantle cell lymphoma. Oncogene: May 20, 2013 (Epub ahead of print). doi: 10.1038/onc.2013.177.

19. Cece R, Barajon I and Tredici G: Cisplatin induces apoptosis in SH-SY5Y human neuroblastoma cell line. Anticancer Res 15 777-782, 1995

20. Zhang YX, Yue Z, Wang PY, et al: Cisplatin upregulates MSH2 expression by reducing miR-21 to inhibit A549 cell growth. Biomed Pharmacother 67: 97-102, 2013.

21. Middlemas DS, Kihl BK, Zhou J and Zhu X: Brain-derived neurotrophic factor promotes survival and chemoprotection of human neuroblastoma cells. J Biol Chem 274: 16451-16460, 1999.

22. Numakawa T, Suzuki S, Kumamaru E, Adachi N, Richards M and Kunugi $\mathrm{H}$ : BDNF function and intracellular signaling in neurons. Histol Histopathol 25: 237-258, 2010.
23. Xiong L, Deng X, Wen Y, Yang Z and Miao X: Association of BDNF and BMPR1A with clinicopathologic parameters in benign and malignant gallbladder lesions. World J Surg Oncol 11: 80, 2013.

24. Garzon R, Calin GA and Croce CM: MicroRNAs in cancer. Annu Rev Med 60: 167-179, 2009.

25. Guo WG, Zhang Y, Ge D, et al: Bioinformatics analyses combined microarray identify the desregulated microRNAs in lung cancer. Eur Rev Med Pharmacol Sci 17: 1509-1516, 2013.

26. Kefas B, Godlewski J, Comeau L, et al: microRNA-7 inhibits the epidermal growth factor receptor and the Akt pathway and is down-regulated in glioblastoma. Cancer Res 68: 3566-3572, 2008.

27. Xiong S, Zheng Y, Jiang P, Liu R, Liu X and Chu Y: MicroRNA-7 inhibits the growth of human non-small cell lung cancer A549 cells through targeting BCL-2. Int J Biol Sci 7: 805-814, 2011.

28. Bhattacharya R, Nicoloso M, Arvizo R, et al: MiR-15a and MiR-16 control Bmi-1 expression in ovarian cancer. Cancer Res 69: 9090-9095, 2009.

29. Bonci D, Coppola V, Musumeci M, et al: The miR-15a-miR-16-1 cluster controls prostate cancer by targeting multiple oncogenic activities. Nat Med 14: 1271-1277, 2008

30. Ma Q, Wang X, Li Z, et al: microRNA-16 represses colorectal cancer cell growth in vitro by regulating the p53/survivin signaling pathway. Oncol Rep 29: 1652-1658, 2013.

31. Lee ST, Chu K, Jung KH, et al: miR-206 regulates brain-derived neurotrophic factor in Alzheimer disease model. Ann Neurol 72: 269-277, 2012.

32. Mellios N, Huang HS, Grigorenko A, Rogaev E and Akbarian S: A set of differentially expressed miRNAs, including miR-30a-5p, act as post-transcriptional inhibitors of BDNF in prefrontal cortex. Hum Mol Genet 17: 3030-3042, 2008.

33. Mellios N, Huang HS, Baker SP, Galdzicka M, Ginns E and Akbarian S: Molecular determinants of dysregulated GABAergic gene expression in the prefrontal cortex of subjects with schizophrenia. Biol Psychiatry 65: 1006-1014, 2009.

34. Imam JS, Plyler JR, Bansal H, et al: Genomic loss of tumor suppressor miRNA-204 promotes cancer cell migration and invasion by activating AKT/mTOR/Racl signaling and actin reorganization. PLoS One 7: e52397, 2012.

35. Xiang Q, Tang H, Yu J, Yin J, Yang X and Lei X: MicroRNA-98 sensitizes cisplatin-resistant human lung adenocarcinoma cells by up-regulation of HMGA2. Pharmazie 68: 274-281, 2013.

36. Chauffert B, Dimanche-Boitrel MT, Garrido C, et al: New insights into the kinetic resistance to anticancer agents. Cytotechnology 27: 225-235, 1998

37. Liu L, Lian J, Zhang H, et al: MicroRNA-302a sensitizes testicular embryonal carcinoma cells to cisplatin-induced cell death. J Cell Physiol: April 27, 2013 (Epub ahead of print). doi: 10.1002/ jcp. 24394

38. Ryan J, Tivnan A, Fay J, et al: MicroRNA-204 increases sensitivity of neuroblastoma cells to cisplatin and is associated with a favourable clinical outcome. Br J Cancer 107: 967-976, 2012. 